\title{
An iterative approach to mixed equilibrium problems and fixed points problems
}

\author{
Yonghong Yao ${ }^{1}$, Yeong-Cheng Liou ${ }^{2}$ and Shin Min Kang ${ }^{3 *}$
}

\section{"Correspondence:}

smkang@gnu.ac.kr

${ }^{3}$ Department of Mathematics and

the RINS, Gyeongsang National

University, Jinju, 660-701, Korea

Full list of author information is

available at the end of the article

\begin{abstract}
In the present paper, an iterative algorithm for solving mixed equilibrium problems and fixed points problems has been constructed. It is shown that under some mild conditions, the sequence generated by the presented algorithm converges strongly to the common solution of mixed equilibrium problems and fixed points problems. As an application, we can find the minimum norm element without involving projection.
\end{abstract}

MSC: 47J05; 47J25; 47H09

Keywords: mixed equilibrium problem; fixed point problem; minimization problem; strictly pseudo-contractive mapping

\section{Introduction}

Let $H$ be a real Hilbert space with the inner product $\langle\cdot, \cdot\rangle$ and the norm $\|\cdot\|$, respectively. Let $C$ be a nonempty closed convex subset of $H$. For a nonlinear mapping $A: C \rightarrow H$ and a bifunction $F: C \times C \rightarrow R$, the mixed equilibrium problem is to find $z \in C$ such that

$$
F(z, y)+\langle A z, y-z\rangle \geq 0, \quad \forall y \in C .
$$

The solution set of (1.1) is denoted by $M E P$. If $A=0$, then (1.1) reduces to the following equilibrium problem of finding $z \in C$ such that

$$
F(z, y) \geq 0, \quad \forall y \in C
$$

The solution set of (1.2) is denoted by $E P$. If $F=0$, then (1.1) reduces to the variational inequality problem of finding $z \in C$ such that

$$
\langle A z, y-z\rangle \geq 0, \quad \forall y \in C .
$$

The solution set of (1.3) is denoted by VI. Problem (1.1) is very general in the sense that it includes, as special cases, optimization problems, variational inequalities, minimax problems, Nash equilibrium problem in noncooperative games and others. See, e.g., [1-22].

For solving mixed equilibrium problem (1.1), Moudafi [9] introduced an iterative algorithm and proved a weak convergence theorem. Further, Takahashi and Takahashi [15]

(c) 2013 Yao et al.: licensee Springer. This is an Open Access article distributed under the terms of the Creative Commons Attribution License (http://creativecommons.org/licenses/by/2.0), which permits unrestricted use, distribution, and reproduction in any medium, provided the original work is properly cited. 
introduced the following iterative algorithm for finding an element of $F(S) \cap M E P$ :

$$
\left\{\begin{array}{l}
F\left(z_{n}, y\right)+\left\langle A x_{n}, y-z_{n}\right\rangle+\frac{1}{\lambda_{n}}\left\langle y-z_{n}, z_{n}-x_{n}\right\rangle \geq 0, \quad \forall y \in C, \\
x_{n+1}=\beta_{n} x_{n}+S\left(\alpha_{n} u+\left(1-\beta_{n}\right) z_{n}\right)
\end{array}\right.
$$

for all $n \geq 0$, where $S: C \rightarrow C$ is a nonexpansive mapping. They proved that the sequence $\left\{x_{n}\right\}$ generated by (1.4) converges strongly to $z=\operatorname{Proj}_{F(S) \cap M E P}(u)$.

Recently, Yao and Shahzad [19] gave the following iteration process for nonexpansive mappings with perturbation: $x_{1} \in C$ and

$$
x_{n+1}=\left(1-\beta_{n}\right) x_{n}+\beta_{n} \operatorname{Proj}_{C}\left(\alpha_{n} u_{n}+\left(1-\alpha_{n}\right) T x_{n}\right), \quad n \geq 0,
$$

where $\left\{\alpha_{n}\right\}$ and $\left\{\beta_{n}\right\}$ are sequences in $[0,1]$, and the sequence $\left\{u_{n}\right\}$ in $H$ is a small perturbation for the $n$-step iteration satisfying $\left\|u_{n}\right\| \rightarrow 0$ as $n \rightarrow \infty$. In fact, there are perturbations always occurring in the iterative processes because the manipulations are inaccurate.

Using the ideas in [19], Chuang et al. [4] introduced the following iteration process for finding a common element of the set of solutions of the equilibrium problem and the set of fixed points for a quasi-nonexpansive mapping with perturbation: $q_{1} \in H$ and

$$
\left\{\begin{array}{l}
x_{n} \in C \text { such that } F\left(x_{n}, y\right)+\frac{1}{\lambda_{n}}\left\langle y-x_{n}, x_{n}-q_{n}\right\rangle \geq 0, \quad \forall y \in C, \\
q_{n+1}=\alpha_{n} u_{n}+\left(1-\alpha_{n}\right)\left(\beta_{n} x_{n}+\left(1-\beta_{n}\right) S x_{n}\right)
\end{array}\right.
$$

for all $n \geq 0$. They showed that the sequence $\left\{q_{n}\right\}$ converges strongly to $\operatorname{Proj}_{F(S) \cap E P}$.

Motivated and inspired by the above works, in the present paper, we construct an iterative algorithm for solving mixed equilibrium problems and fixed points problems. It is shown that under some mild conditions the sequence $\left\{x_{n}\right\}$ generated by the presented algorithm converges strongly to the common solution of mixed equilibrium problems and fixed points problems. As an application, we can find the minimum norm element without involving projection.

\section{Preliminaries}

Let $C$ be a nonempty closed convex subset of a real Hilbert space $H$. Recall that a mapping $A: C \rightarrow H$ is called $\alpha$-inverse-strongly monotone if there exists a positive real number $\alpha>0$ such that

$$
\langle A x-A y, x-y\rangle \geq \alpha\|A x-A y\|^{2}, \quad \forall x, y \in C .
$$

It is clear that any $\alpha$-inverse-strongly monotone mapping is monotone and $\frac{1}{\alpha}$-Lipschitz continuous. A mapping $S: C \rightarrow C$ is said to be nonexpansive if $\|S x-S y\| \leq\|x-y\|$ for all $x, y \in C$. And a mapping $S: C \rightarrow C$ is said to be strictly pseudo-contractive if there exists a constant $0 \leq \kappa<1$ such that

$$
\|S x-S y\|^{2} \leq\|x-y\|^{2}+\kappa\|(I-S) x-(I-S) y\|^{2}, \quad \forall x, y \in C .
$$

For such a case, we also say that $S$ is a $\kappa$-strictly pseudo-contractive mapping.

Throughout this paper, we assume that a bifunction $F: C \times C \rightarrow R$ satisfies the following conditions: 
(H1) $F(x, x)=0$ for all $x \in C$;

(H2) $F$ is monotone, i.e., $F(x, y)+F(y, x) \leq 0$ for all $x, y \in C$;

(H3) for each $x, y, z \in C, \lim _{t \downarrow 0} F(t z+(1-t) x, y) \leq F(x, y)$;

(H4) for each $x \in C, y \mapsto F(x, y)$ is convex and lower semicontinuous.

We need the following lemmas for proving our main results.

Lemma 2.1 [7] Let $C$ be a nonempty closed convex subset of a real Hilbert space H. Let $F: C \times C \rightarrow R$ be a bifunction which satisfies conditions (H1)-(H4). Let $r>0$ and $x \in H$. Then there exists $z \in C$ such that

$$
F(z, y)+\frac{1}{r}\langle y-z, z-x\rangle \geq 0, \quad \forall y \in C .
$$

Further, if $T_{r}(x)=\left\{z \in C: F(z, y)+\frac{1}{r}\langle y-z, z-x\rangle \geq 0, \forall y \in C\right\}$, then we have

(i) $T_{r}$ is single-valued and $T_{r}$ is firmly nonexpansive, i.e., for any $x, y \in H$, $\left\|T_{r} x-T_{r} y\right\|^{2} \leq\left\langle T_{r} x-T_{r} y, x-y\right\rangle$

(ii) $E P$ is closed and convex and $E P=F\left(T_{r}\right)$.

Lemma 2.2 [19] Let C, H, F and $T_{r} x$ be as in Lemma 2.1. Then we have

$$
\left\|T_{s} x-T_{t} x\right\|^{2} \leq \frac{s-t}{s}\left\langle T_{s} x-T_{t} x, T_{s} x-x\right\rangle
$$

for all $s, t>0$ and $x \in H$.

Lemma 2.3 [19] Let $C$ be a nonempty closed convex subset of a real Hilbert space $H$. Let the mapping $A: C \rightarrow H$ be $\alpha$-inverse strongly monotone and $r>0$ be a constant. Then we have

$$
\|(I-r A) x-(I-r A) y\|^{2} \leq\|x-y\|^{2}+r(r-2 \alpha)\|A x-A y\|^{2}, \quad \forall x, y \in C .
$$

In particular, if $0 \leq r \leq 2 \alpha$, then $I-r A$ is nonexpansive.

Lemma 2.4 [23] Let $\left\{x_{n}\right\}$ and $\left\{y_{n}\right\}$ be bounded sequences in a Banach space $X$ and let $\left\{\beta_{n}\right\}$ be a sequence in $[0,1]$ with $0<\liminf _{n \rightarrow \infty} \beta_{n} \leq \lim \sup _{n \rightarrow \infty} \beta_{n}<1$. Suppose that $x_{n+1}=(1-$ $\left.\beta_{n}\right) y_{n}+\beta_{n} x_{n}$ for all $n \geq 0$ and $\lim \sup _{n \rightarrow \infty}\left(\left\|y_{n+1}-y_{n}\right\|-\left\|x_{n+1}-x_{n}\right\|\right) \leq 0$. Then $\lim _{n \rightarrow \infty} \| y_{n}-$ $x_{n} \|=0$.

Lemma 2.5 [24] Let $C$ be a nonempty closed convex subset of a real Hilbert space H. Let $S: C \rightarrow C$ be a $\lambda$-strict pseudo-contraction. Then we have

(i) $F(S)=\{x: S x=x\}$ is closed convex;

(ii) $\kappa I+(1-\kappa) S$ for $\kappa \in[\lambda, 1)$ is nonexpansive.

Lemma 2.6 [25] Let $C$ be a nonempty closed and convex of a real Hilbert space H. Let $S: C \rightarrow C$ be a $\kappa$-strictly pseudo-contractive mapping. Then $I-S$ is demi-closed at 0 , i.e., if $x_{n} \rightarrow x \in C$ and $x_{n}-S x_{n} \rightarrow 0$, then $x=S x$.

Lemma $2.7[16]$ Assume that $\left\{a_{n}\right\}$ is a sequence of nonnegative real numbers such that

$$
a_{n+1} \leq\left(1-\gamma_{n}\right) a_{n}+\delta_{n} \gamma_{n}
$$


where $\left\{\gamma_{n}\right\}$ is a sequence in $(0,1)$ and $\left\{\delta_{n}\right\}$ is a sequence such that

(1) $\sum_{n=1}^{\infty} \gamma_{n}=\infty$;

(2) $\lim \sup _{n \rightarrow \infty} \delta_{n} \leq 0$ or $\sum_{n=1}^{\infty}\left|\delta_{n} \gamma_{n}\right|<\infty$.

Then $\lim _{n \rightarrow \infty} a_{n}=0$.

\section{Main results}

In this section, we prove our main results.

Theorem 3.1 Let $C$ be a nonempty closed convex subset of a real Hilbert space $H$ and let $F$ : $C \times C \rightarrow R$ be a bifunction satisfying conditions $(\mathrm{H} 1)-(\mathrm{H} 4)$. Let $A: C \rightarrow H$ be an $\alpha$-inversestrongly monotone mapping and let $S: C \rightarrow C$ be a $\kappa$-strictly pseudo-contractive mapping. Suppose that $F(S) \cap M E P \neq \emptyset$. Let $x_{0} \in C,\left\{z_{n}\right\}$ and $\left\{x_{n}\right\}$ be sequences in $C$ generated by

$$
\left\{\begin{array}{l}
F\left(z_{n}, y\right)+\left\langle A x_{n}, y-z_{n}\right\rangle+\frac{1}{\lambda_{n}}\left\langle y-z_{n}, z_{n}-\left(\alpha_{n} u_{n}+\left(1-\alpha_{n}\right) x_{n}\right)\right\rangle \geq 0, \quad \forall y \in C, \\
x_{n+1}=\beta_{n} x_{n}+\left(1-\beta_{n}\right) \gamma z_{n}+\left(1-\beta_{n}\right)(1-\gamma) S z_{n},
\end{array}\right.
$$

for all $n \geq 0$, where $\left\{\lambda_{n}\right\} \subset(0,2 \alpha),\left\{\alpha_{n}\right\} \subset(0,1)$ and $\left\{\beta_{n}\right\} \subset(0,1)$ satisfy

(r1) $\lim _{n \rightarrow \infty} u_{n}=u$ for some $u \in H$

(r2) $\lim _{n \rightarrow \infty} \alpha_{n}=0$ and $\sum_{n=1}^{\infty} \alpha_{n}=\infty$;

(r3) $0<c \leq \beta_{n} \leq d<1$ and $\gamma \in[\kappa, 1)$;

(r4) $a\left(1-\alpha_{n}\right) \leq \lambda_{n} \leq b\left(1-\alpha_{n}\right)$, where $[a, b] \subset(0,2 \alpha)$ and $\lim _{n \rightarrow \infty}\left(\lambda_{n+1}-\lambda_{n}\right)=0$.

Then $\left\{x_{n}\right\}$ generated by (3.1) converges strongly to $\operatorname{Proj}_{F(S) \cap M E P}(u)$.

Proof Note that $z_{n}$ can be rewritten as $z_{n}=T_{\lambda_{n}}\left(\alpha_{n} u_{n}+\left(1-\alpha_{n}\right) x_{n}-\lambda_{n} A x_{n}\right)$ for each $n$. Take $z \in F(S) \cap M E P$. It is obvious that $z=T_{\lambda_{n}}\left(z-\lambda_{n} A z\right)=T_{\lambda_{n}}\left(\alpha_{n} z+\left(1-\alpha_{n}\right)\left(z-\frac{\lambda_{n} A z}{1-\alpha_{n}}\right)\right)$ for all $n \geq 0$. By using the nonexpansivity of $T_{\lambda_{n}}$ and the convexity of $\|\cdot\|$, we derive

$$
\begin{aligned}
& \left\|z_{n}-z\right\|^{2} \\
& \quad=\left\|T_{\lambda_{n}}\left(\alpha_{n} u_{n}+\left(1-\alpha_{n}\right) x_{n}-\lambda_{n} A x_{n}\right)-T_{\lambda_{n}}\left(z-\lambda_{n} A z\right)\right\|^{2} \\
& \quad=\left\|T_{\lambda_{n}}\left(\alpha_{n} u_{n}+\left(1-\alpha_{n}\right)\left(x_{n}-\frac{\lambda_{n} A x_{n}}{1-\alpha_{n}}\right)\right)-T_{\lambda_{n}}\left(\alpha_{n} z+\left(1-\alpha_{n}\right)\left(z-\frac{\lambda_{n} A z}{1-\alpha_{n}}\right)\right)\right\|^{2} \\
& \quad \leq\left\|\left(\alpha_{n} u_{n}+\left(1-\alpha_{n}\right)\left(x_{n}-\frac{\lambda_{n} A x_{n}}{1-\alpha_{n}}\right)\right)-\left(\alpha_{n} z+\left(1-\alpha_{n}\right)\left(z-\frac{\lambda_{n} A z}{1-\alpha_{n}}\right)\right)\right\|^{2} \\
& \quad=\left\|\left(1-\alpha_{n}\right)\left(\left(x_{n}-\frac{\lambda_{n} A x_{n}}{1-\alpha_{n}}\right)-\left(z-\frac{\lambda_{n} A z}{1-\alpha_{n}}\right)\right)+\alpha_{n}\left(u_{n}-z\right)\right\|^{2} \\
& \quad \leq\left(1-\alpha_{n}\right)\left\|\left(x_{n}-\frac{\lambda_{n} A x_{n}}{1-\alpha_{n}}\right)-\left(z-\frac{\lambda_{n} A z}{1-\alpha_{n}}\right)\right\|^{2}+\alpha_{n}\left\|u_{n}-z\right\|^{2} .
\end{aligned}
$$

Since $A$ is $\alpha$-inverse strongly monotone, we know from Lemma 2.3 that

$$
\begin{aligned}
& \left\|\left(x_{n}-\frac{\lambda_{n} A x_{n}}{1-\alpha_{n}}\right)-\left(z-\frac{\lambda_{n} A z}{1-\alpha_{n}}\right)\right\|^{2} \\
& \quad \leq\left\|x_{n}-z\right\|^{2}+\frac{\lambda_{n}\left(\lambda_{n}-2\left(1-\alpha_{n}\right) \alpha\right)}{\left(1-\alpha_{n}\right)^{2}}\left\|A x_{n}-A z\right\|^{2} .
\end{aligned}
$$


It follows that

$$
\begin{aligned}
\left\|z_{n}-z\right\|^{2} & \leq\left(1-\alpha_{n}\right)\left(\left\|x_{n}-z\right\|^{2}+\frac{\lambda_{n}\left(\lambda_{n}-2\left(1-\alpha_{n}\right) \alpha\right)}{\left(1-\alpha_{n}\right)^{2}}\left\|A x_{n}-A z\right\|^{2}\right)+\alpha_{n}\left\|u_{n}-z\right\|^{2} \\
& \leq\left(1-\alpha_{n}\right)\left\|x_{n}-z\right\|^{2}+\alpha_{n}\left\|u_{n}-z\right\|^{2} .
\end{aligned}
$$

So, we have

$$
\begin{aligned}
\left\|x_{n+1}-z\right\|^{2} & =\left\|\beta_{n}\left(x_{n}-z\right)+\left(1-\beta_{n}\right)\left((\gamma I+(1-\gamma) S) z_{n}-z\right)\right\|^{2} \\
& \leq \beta_{n}\left\|x_{n}-z\right\|^{2}+\left(1-\beta_{n}\right)\left\|z_{n}-z\right\|^{2} \\
& \leq \beta_{n}\left\|x_{n}-z\right\|^{2}+\left(1-\beta_{n}\right)\left(\left(1-\alpha_{n}\right)\left\|x_{n}-z\right\|^{2}+\alpha_{n}\left\|u_{n}-z\right\|^{2}\right) \\
& =\left[1-\left(1-\beta_{n}\right) \alpha_{n}\right]\left\|x_{n}-z\right\|^{2}+\left(1-\beta_{n}\right) \alpha_{n}\left\|u_{n}-z\right\|^{2} \\
& \leq \max \left\{\left\|x_{n}-z\right\|^{2},\left\|u_{n}-z\right\|^{2}\right\} .
\end{aligned}
$$

Since $\lim _{n \rightarrow \infty} u_{n}=u,\left\{u_{n}\right\}$ is bounded. Therefore, by induction, we deduce that $\left\{x_{n}\right\}$ is bounded. Hence, $\left\{A x_{n}\right\},\left\{z_{n}\right\}$ and $\left\{S z_{n}\right\}$ are also bounded.

Putting $y_{n}=\alpha_{n} u_{n}+\left(1-\alpha_{n}\right) x_{n}-\lambda_{n} A x_{n}$ for all $n$, we have

$$
z_{n+1}-z_{n}=T_{\lambda_{n+1}} y_{n+1}-T_{\lambda_{n+1}} y_{n}+T_{\lambda_{n+1}} y_{n}-T_{\lambda_{n}} y_{n} .
$$

It follows that

$$
\begin{aligned}
\left\|z_{n+1}-z_{n}\right\| & \leq\left\|T_{\lambda_{n+1}} y_{n+1}-T_{\lambda_{n+1}} y_{n}\right\|+\left\|T_{\lambda_{n+1}} y_{n}-T_{\lambda_{n}} y_{n}\right\| \\
& \leq\left\|y_{n+1}-y_{n}\right\|+\left\|T_{\lambda_{n+1}} y_{n}-T_{\lambda_{n}} y_{n}\right\| .
\end{aligned}
$$

From Lemma 2.3, we know that $I-\lambda A$ is nonexpansive for all $\lambda \in(0,2 \alpha)$. Thus, we have $I-\frac{\lambda_{n+1}}{1-\alpha_{n+1}} A$ is nonexpansive for all $n$ due to the fact that $\frac{\lambda_{n+1}}{1-\alpha_{n+1}} \in(0,2 \alpha)$. Then we get

$$
\begin{aligned}
\left\|y_{n+1}-y_{n}\right\|= & \left\|\alpha_{n+1} u_{n+1}+\left(1-\alpha_{n+1}\right) x_{n+1}-\lambda_{n+1} A x_{n+1}-\left(\alpha_{n} u_{n}+\left(1-\alpha_{n}\right) x_{n}-\lambda_{n} A x_{n}\right)\right\| \\
\leq & \left\|\left(1-\alpha_{n+1}\right)\left(x_{n+1}-\frac{\lambda_{n+1}}{1-\alpha_{n+1}} A x_{n+1}\right)-\left(1-\alpha_{n}\right)\left(x_{n}-\frac{\lambda_{n}}{1-\alpha_{n}} A x_{n}\right)\right\| \\
& +\alpha_{n+1}\left\|u_{n+1}\right\|+\alpha_{n}\left\|u_{n}\right\| \\
\leq & \left(1-\alpha_{n+1}\right)\left\|\left(I-\frac{\lambda_{n+1}}{1-\alpha_{n+1}} A\right) x_{n+1}-\left(I-\frac{\lambda_{n+1}}{1-\alpha_{n+1}} A\right) x_{n}\right\| \\
& +\left\|\left(1-\alpha_{n+1}\right)\left(x_{n}-\frac{\lambda_{n+1}}{1-\alpha_{n+1}} A x_{n}\right)-\left(1-\alpha_{n}\right)\left(x_{n}-\frac{\lambda_{n}}{1-\alpha_{n}} A x_{n}\right)\right\| \\
& +\alpha_{n+1}\left\|u_{n+1}\right\|+\alpha_{n}\left\|u_{n}\right\| \\
\leq & \left\|x_{n+1}-x_{n}\right\|+\left|\alpha_{n+1}-\alpha_{n}\right|\left\|x_{n}\right\|+\left|\lambda_{n+1}-\lambda_{n}\right|\left\|A x_{n}\right\| \\
& +\alpha_{n+1}\left\|u_{n+1}\right\|+\alpha_{n}\left\|u_{n}\right\| .
\end{aligned}
$$

By Lemma 2.2, we have

$$
\left\|T_{\lambda_{n+1}} y_{n}-T_{\lambda_{n}} y_{n}\right\| \leq \frac{\left|\lambda_{n+1}-\lambda_{n}\right|}{\lambda_{n+1}}\left\|T_{\lambda_{n+1}} y_{n}-y_{n}\right\| .
$$


From (3.3)-(3.5), we obtain

$$
\begin{aligned}
\left\|z_{n+1}-z_{n}\right\| \leq & \left\|x_{n+1}-x_{n}\right\|+\left|\alpha_{n+1}-\alpha_{n}\right|\left\|x_{n}\right\|+\left|\lambda_{n+1}-\lambda_{n}\right|\left\|A x_{n}\right\| \\
& +\frac{\left|\lambda_{n+1}-\lambda_{n}\right|}{\lambda_{n+1}}\left\|T_{\lambda_{n+1}} y_{n}-y_{n}\right\|+\alpha_{n+1}\left\|u_{n+1}\right\|+\alpha_{n}\left\|u_{n}\right\| .
\end{aligned}
$$

Then

$$
\begin{aligned}
& \left\|(\gamma I+(1-\gamma) S) z_{n+1}-(\gamma I+(1-\gamma) S) z_{n}\right\| \\
& \leq\left\|z_{n+1}-z_{n}\right\| \\
& \leq\left\|x_{n+1}-x_{n}\right\|+\left|\alpha_{n+1}-\alpha_{n}\right|\left\|x_{n}\right\|+\left|\lambda_{n+1}-\lambda_{n}\right|\left\|A x_{n}\right\| \\
& \quad+\frac{\left|\lambda_{n+1}-\lambda_{n}\right|}{\lambda_{n+1}}\left\|T_{\lambda_{n+1}} y_{n}-y_{n}\right\|+\alpha_{n+1}\left\|u_{n+1}\right\|+\alpha_{n}\left\|u_{n}\right\| .
\end{aligned}
$$

Therefore,

$$
\begin{aligned}
& \left\|(\gamma I+(1-\gamma) S) z_{n+1}-(\gamma I+(1-\gamma) S) z_{n}\right\|-\left\|x_{n+1}-x_{n}\right\| \\
& \quad \leq\left|\alpha_{n+1}-\alpha_{n}\right|\left\|x_{n}\right\|+\left|\lambda_{n+1}-\lambda_{n}\right|\left\|A x_{n}\right\|+\alpha_{n+1}\left\|u_{n+1}\right\|+\alpha_{n}\left\|u_{n}\right\| \\
& \quad+\frac{\left|\lambda_{n+1}-\lambda_{n}\right|}{\lambda_{n+1}}\left\|T_{\lambda_{n+1}} y_{n}-y_{n}\right\| .
\end{aligned}
$$

Since $\alpha_{n} \rightarrow 0, \lambda_{n+1}-\lambda_{n} \rightarrow 0$ and $\liminf _{n \rightarrow \infty} \lambda_{n}>0$, we obtain

$$
\limsup _{n \rightarrow \infty}\left(\left\|(\gamma I+(1-\gamma) S) z_{n+1}-(\gamma I+(1-\gamma) S) z_{n}\right\|-\left\|x_{n+1}-x_{n}\right\|\right) \leq 0
$$

This together with Lemma 2.4 implies that

$$
\lim _{n \rightarrow \infty}\left\|(\gamma I+(1-\gamma) S) z_{n}-x_{n}\right\|=0
$$

Consequently, we obtain

$$
\lim _{n \rightarrow \infty}\left\|x_{n+1}-x_{n}\right\|=\lim _{n \rightarrow \infty}\left(1-\beta_{n}\right)\left\|(\gamma I+(1-\gamma) S) z_{n}-x_{n}\right\|=0 .
$$

From (3.1) and (3.2), we have

$$
\begin{aligned}
\left\|x_{n+1}-z\right\|^{2} \leq & \left(1-\beta_{n}\right)\left\|(\gamma I+(1-\gamma) S) T_{\lambda_{n}}\left(\alpha_{n} u_{n}+\left(1-\alpha_{n}\right) x_{n}-\lambda_{n} A x_{n}\right)-z\right\|^{2} \\
& +\beta_{n}\left\|x_{n}-z\right\|^{2} \\
\leq & \left(1-\beta_{n}\right)\left\{\left(1-\alpha_{n}\right)\left(\left\|x_{n}-z\right\|^{2}+\frac{\lambda_{n}}{\left(1-\alpha_{n}\right)^{2}}\left(\lambda_{n}-2\left(1-\alpha_{n}\right) \alpha\right)\left\|A x_{n}-A z\right\|^{2}\right)\right. \\
& \left.+\alpha_{n}\left\|u_{n}-z\right\|^{2}\right\}+\beta_{n}\left\|x_{n}-z\right\|^{2} \\
& \left(1-\left(1-\beta_{n}\right) \alpha_{n}\right)\left\|x_{n}-z\right\|^{2}+\frac{\left(1-\beta_{n}\right) \lambda_{n}}{1-\alpha_{n}}\left(\lambda_{n}-2\left(1-\alpha_{n}\right) \alpha\right)\left\|A x_{n}-A z\right\|^{2} \\
= & +\left(1-\beta_{n}\right) \alpha_{n}\left\|u_{n}-z\right\|^{2}
\end{aligned}
$$




$$
\begin{aligned}
\leq & \left\|x_{n}-z\right\|^{2}+\frac{\left(1-\beta_{n}\right) \lambda_{n}}{1-\alpha_{n}}\left(\lambda_{n}-2\left(1-\alpha_{n}\right) \alpha\right)\left\|A x_{n}-A z\right\|^{2} \\
& +\left(1-\beta_{n}\right) \alpha_{n}\left\|u_{n}-z\right\|^{2} .
\end{aligned}
$$

Then we obtain

$$
\begin{aligned}
& \frac{\left(1-\beta_{n}\right) \lambda_{n}}{1-\alpha_{n}}\left(2\left(1-\alpha_{n}\right) \alpha-\lambda_{n}\right)\left\|A x_{n}-A z\right\|^{2} \\
& \quad \leq\left\|x_{n}-z\right\|^{2}-\left\|x_{n+1}-z\right\|^{2}+\left(1-\beta_{n}\right) \alpha_{n}\left\|u_{n}-z\right\|^{2} \\
& \quad \leq\left(\left\|x_{n}-z\right\|-\left\|x_{n+1}-z\right\|\right)\left\|x_{n+1}-x_{n}\right\|+\left(1-\beta_{n}\right) \alpha_{n}\left\|u_{n}-z\right\|^{2} .
\end{aligned}
$$

Since $\lim _{n \rightarrow \infty} \alpha_{n}=0, \lim _{n \rightarrow \infty}\left\|x_{n+1}-x_{n}\right\|=0$ and $\liminf _{n \rightarrow \infty} \frac{\left(1-\beta_{n}\right) \lambda_{n}}{1-\alpha_{n}}\left(2\left(1-\alpha_{n}\right) \alpha-\lambda_{n}\right)>0$, we have

$$
\lim _{n \rightarrow \infty}\left\|A x_{n}-A z\right\|=0
$$

Next, we show $\left\|x_{n}-z_{n}\right\|=\left\|x_{n}-T_{\lambda_{n}} y_{n}\right\| \rightarrow 0$. By using the firm nonexpansivity of $T_{\lambda_{n}}$, we have

$$
\begin{aligned}
\left\|T_{\lambda_{n}} y_{n}-z\right\|^{2}= & \left\|T_{\lambda_{n}} y_{n}-T_{\lambda_{n}}\left(z-\lambda_{n} A z\right)\right\|^{2} \\
\leq & \left\langle y_{n}-\left(z-\lambda_{n} A z\right), T_{\lambda_{n}} y_{n}-z\right\rangle \\
= & \frac{1}{2}\left(\left\|y_{n}-\left(z-\lambda_{n} A z\right)\right\|^{2}+\left\|T_{\lambda_{n}} y_{n}-z\right\|^{2}\right. \\
& \left.-\left\|\alpha_{n} u_{n}+\left(1-\alpha_{n}\right) x_{n}-\lambda_{n}\left(A x_{n}-\lambda_{n} A z\right)-T_{\lambda_{n}} y_{n}\right\|^{2}\right) .
\end{aligned}
$$

We note that

$$
\left\|y_{n}-\left(z-\lambda_{n} A z\right)\right\|^{2} \leq\left(1-\alpha_{n}\right)\left\|x_{n}-z\right\|^{2}+\alpha_{n}\left\|u_{n}-z\right\|^{2} .
$$

Thus,

$$
\begin{aligned}
\left\|T_{\lambda_{n}} y_{n}-z\right\|^{2} \leq & \frac{1}{2}\left(\left(1-\alpha_{n}\right)\left\|x_{n}-z\right\|^{2}+\alpha_{n}\left\|u_{n}-z\right\|^{2}+\left\|T_{\lambda_{n}} y_{n}-z\right\|^{2}\right. \\
& \left.-\left\|\alpha_{n} u_{n}+\left(1-\alpha_{n}\right) x_{n}-T_{\lambda_{n}} y_{n}-\lambda_{n}\left(A x_{n}-\lambda_{n} A z\right)\right\|^{2}\right) .
\end{aligned}
$$

That is,

$$
\begin{aligned}
\left\|T_{\lambda_{n}} y_{n}-z\right\|^{2} \leq & \left(1-\alpha_{n}\right)\left\|x_{n}-z\right\|^{2}+\alpha_{n}\left\|u_{n}-z\right\|^{2} \\
& -\left\|\alpha_{n} u_{n}+\left(1-\alpha_{n}\right) x_{n}-T_{\lambda_{n}} y_{n}-\lambda_{n}\left(A x_{n}-\lambda_{n} A z\right)\right\|^{2} \\
= & \left(1-\alpha_{n}\right)\left\|x_{n}-z\right\|^{2}+\alpha_{n}\left\|u_{n}-z\right\|^{2}-\left\|\alpha_{n} u_{n}+\left(1-\alpha_{n}\right) x_{n}-T_{\lambda_{n}} y_{n}\right\|^{2} \\
& +2 \lambda_{n}\left(\alpha_{n} u_{n}+\left(1-\alpha_{n}\right) x_{n}-T_{\lambda_{n}} y_{n}, A x_{n}-A z\right\rangle-\lambda_{n}^{2}\left\|A x_{n}-A z\right\|^{2} \\
\leq & \left(1-\alpha_{n}\right)\left\|x_{n}-z\right\|^{2}+\alpha_{n}\left\|u_{n}-z\right\|^{2}-\left\|\alpha_{n} u_{n}+\left(1-\alpha_{n}\right) x_{n}-T_{\lambda_{n}} y_{n}\right\|^{2} \\
& +2 \lambda_{n}\left\|\alpha_{n} u_{n}+\left(1-\alpha_{n}\right) x_{n}-T_{\lambda_{n}} y_{n}\right\|\left\|A x_{n}-A z\right\| .
\end{aligned}
$$


It follows that

$$
\begin{aligned}
\left\|x_{n+1}-z\right\|^{2} \leq & \beta_{n}\left\|x_{n}-z\right\|^{2}+\left(1-\beta_{n}\right)\left(1-\alpha_{n}\right)\left\|x_{n}-z\right\|^{2}+\left(1-\beta_{n}\right) \alpha_{n}\left\|u_{n}-z\right\|^{2} \\
& -\left(1-\beta_{n}\right)\left\|\alpha_{n} u_{n}+\left(1-\alpha_{n}\right) x_{n}-T_{\lambda_{n}} y_{n}\right\|^{2} \\
& +2 \lambda_{n}\left(1-\beta_{n}\right)\left\|\alpha_{n} u_{n}+\left(1-\alpha_{n}\right) x_{n}-T_{\lambda_{n}} y_{n}\right\|\left\|A x_{n}-A z\right\| \\
= & \left(1-\left(1-\beta_{n}\right) \alpha_{n}\right)\left\|x_{n}-z\right\|^{2}+\left(1-\beta_{n}\right) \alpha_{n}\left\|u_{n}-z\right\|^{2} \\
& -\left(1-\beta_{n}\right)\left\|\alpha_{n} u_{n}+\left(1-\alpha_{n}\right) x_{n}-T_{\lambda_{n}} y_{n}\right\|^{2} \\
& +2 \lambda_{n}\left(1-\beta_{n}\right)\left\|\alpha_{n} u_{n}+\left(1-\alpha_{n}\right) x_{n}-T_{\lambda_{n}} y_{n}\right\|\left\|A x_{n}-A z\right\| .
\end{aligned}
$$

Hence,

$$
\begin{aligned}
(1- & \left.\beta_{n}\right)\left\|\alpha_{n} u_{n}+\left(1-\alpha_{n}\right) x_{n}-T_{\lambda_{n}} y_{n}\right\|^{2} \\
\leq & \left\|x_{n}-z\right\|^{2}-\left\|x_{n+1}-z\right\|^{2}-\left(1-\beta_{n}\right) \alpha_{n}\left\|x_{n}-z\right\|^{2} \\
& +\left(1-\beta_{n}\right) \alpha_{n}\left\|u_{n}-z\right\|^{2}+2 \lambda_{n}\left(1-\beta_{n}\right)\left\|\alpha_{n} u_{n}+\left(1-\alpha_{n}\right) x_{n}-T_{\lambda_{n}} y_{n}\right\|\left\|A x_{n}-A z\right\| \\
\leq & \left(\left\|x_{n}-z\right\|+\left\|x_{n+1}-z\right\|\right)\left\|x_{n+1}-x_{n}\right\|+\left(1-\beta_{n}\right) \alpha_{n}\left\|u_{n}-z\right\|^{2} \\
& +2 \lambda_{n}\left(1-\beta_{n}\right)\left\|\alpha_{n} u_{n}+\left(1-\alpha_{n}\right) x_{n}-T_{\lambda_{n}} y_{n}\right\|\left\|A x_{n}-A z\right\| .
\end{aligned}
$$

Since lim $\sup _{n \rightarrow \infty} \beta_{n}<1,\left\|x_{n+1}-x_{n}\right\| \rightarrow 0, \alpha_{n} \rightarrow 0$ and $\left\|A x_{n}-A z\right\| \rightarrow 0$, we deduce

$$
\lim _{n \rightarrow \infty}\left\|\alpha_{n} u_{n}+\left(1-\alpha_{n}\right) x_{n}-T_{\lambda_{n}} y_{n}\right\|=0
$$

This implies that

$$
\lim _{n \rightarrow \infty}\left\|x_{n}-z_{n}\right\|=\left\|x_{n}-T_{\lambda_{n}} y_{n}\right\|=0 .
$$

Put $\tilde{x}=\operatorname{Proj}_{F(S) \cap M E P}(u)$. We will finally show that $x_{n} \rightarrow \tilde{x}$.

Setting $v_{n}=x_{n}-\frac{\lambda_{n}}{1-\alpha_{n}}\left(A x_{n}-A \tilde{x}\right)$ for all $n$. Taking $z=\tilde{x}$ in (3.7) to get $\left\|A x_{n}-A \tilde{x}\right\| \rightarrow 0$. First, we prove $\lim \sup _{n \rightarrow \infty}\left\langle u-\tilde{x}, v_{n}-\tilde{x}\right\rangle \leq 0$. We take a subsequence $\left\{v_{n_{i}}\right\}$ of $\left\{v_{n}\right\}$ such that

$$
\limsup _{n \rightarrow \infty}\left\langle u-\tilde{x}, v_{n}-\tilde{x}\right\rangle=\lim _{i \rightarrow \infty}\left\langle u-\tilde{x}, v_{n_{i}}-\tilde{x}\right\rangle .
$$

It is clear that $\left\{v_{n_{i}}\right\}$ is bounded due to the boundedness of $\left\{x_{n}\right\}$ and $\left\|A x_{n}-A \tilde{x}\right\| \rightarrow 0$. Then there exists a subsequence $\left\{v_{n_{i_{j}}}\right\}$ of $\left\{v_{n_{i}}\right\}$ which converges weakly to some point $w \in C$. Hence, $\left\{x_{n_{i_{j}}}\right\}$ also converges weakly to $w$. At the same time, from (3.6) and (3.8), we have

$$
\lim _{j \rightarrow \infty}\left\|x_{n_{i_{j}}}-(\gamma I+(1-\gamma) S) x_{n_{i_{j}}}\right\|=0
$$

By the demi-closedness principle (see Lemma 2.6) and (3.9), we deduce $w \in F(S)$.

Further, we show that $w$ is also in MEP. From (3.1), we have

$$
F\left(z_{n}, y\right)+\left\langle A x_{n}, y-z_{n}\right\rangle+\frac{1}{\lambda_{n}}\left\langle y-z_{n}, z_{n}-\left(\alpha_{n} u_{n}+\left(1-\alpha_{n}\right) x_{n}\right)\right\rangle \geq 0 .
$$


From (H2), we have

$$
\left\langle A x_{n}, y-z_{n}\right\rangle+\frac{1}{\lambda_{n}}\left\langle y-z_{n}, z_{n}-\left(\alpha_{n} u_{n}+\left(1-\alpha_{n}\right) x_{n}\right)\right\rangle \geq F\left(y, z_{n}\right)
$$

Put $x_{t}=t y+(1-t) w$ for all $t \in\left(0,1-\frac{\lambda}{2 \alpha}\right)$ and $y \in C$. Then we have $x_{t} \in C$. So, from (3.10), we have

$$
\begin{aligned}
\left\langle x_{t}-z_{n}, A x_{t}\right\rangle \geq & \left\langle x_{t}-z_{n}, A x_{t}\right\rangle-\left\langle x_{t}-z_{n}, A x_{n}\right\rangle \\
& -\frac{1}{\lambda_{n}}\left\langle x_{t}-z_{n}, z_{n}-\left(\alpha_{n} u_{n}+\left(1-\alpha_{n}\right) x_{n}\right)\right\rangle+F\left(x_{t}, z_{n}\right) \\
= & \left\langle x_{t}-z_{n}, A x_{t}-A z_{n}\right\rangle+\left\langle x_{t}-z_{n}, A z_{n}-A x_{n}\right\rangle \\
& -\frac{1}{\lambda_{n}}\left\langle x_{t}-z_{n}, z_{n}-\left(\alpha_{n} u_{n}+\left(1-\alpha_{n}\right) x_{n}\right)\right\rangle+F\left(x_{t}, z_{n}\right) .
\end{aligned}
$$

Since $\left\|z_{n}-x_{n}\right\| \rightarrow 0$, we have $\left\|A z_{n}-A x_{n}\right\| \rightarrow 0$. Further, from monotonicity of $A$, we have $\left\langle x_{t}-z_{n}, A x_{t}-A z_{n}\right\rangle \geq 0$. So, from (H4), we have

$$
\left\langle x_{t}-w, A x_{t}\right\rangle \geq F\left(x_{t}, w\right), \quad \text { as } n \rightarrow \infty \text {. }
$$

From (H1), (H4) and (3.11), we also have

$$
\begin{aligned}
0 & =F\left(x_{t}, x_{t}\right) \\
& \leq t F\left(x_{t}, y\right)+(1-t) F\left(x_{t}, w\right) \\
& \leq t F\left(x_{t}, y\right)+(1-t)\left\langle x_{t}-w, A x_{t}\right\rangle \\
& =t F\left(x_{t}, y\right)+(1-t) t\left\langle y-w, A x_{t}\right\rangle
\end{aligned}
$$

and hence

$$
0 \leq F\left(x_{t}, y\right)+(1-t)\left\langle y-w, A x_{t}\right\rangle
$$

Letting $t \rightarrow 0$, we have, for each $y \in C$,

$$
0 \leq F(w, y)+\langle y-w, A w\rangle
$$

This implies $w \in M E P$. Hence, we have $w \in F(S) \cap M E P$. This implies that

$$
\limsup _{n \rightarrow \infty}\left\langle u-\tilde{x}, v_{n}-\tilde{x}\right\rangle=\lim _{j \rightarrow \infty}\left\langle u-\tilde{x}, v_{n_{i_{j}}}-\tilde{x}\right\rangle=\langle u-\tilde{x}, w-\tilde{x}\rangle .
$$

Note that $\tilde{x}=\operatorname{Proj}_{F(S) \cap M E P}(u)$. Then $\langle u-\tilde{x}, w-\tilde{x}\rangle \leq 0, w \in F(S) \cap M E P$. Therefore,

$$
\limsup _{n \rightarrow \infty}\left\langle u-\tilde{x}, v_{n}-\tilde{x}\right\rangle \leq 0 .
$$

Since $u_{n} \rightarrow u$, we have

$$
\limsup _{n \rightarrow \infty}\left\langle u_{n}-\tilde{x}, v_{n}-\tilde{x}\right\rangle \leq 0
$$


From (3.1), we have

$$
\begin{aligned}
& \left\|x_{n+1}-\tilde{x}\right\|^{2} \\
& \leq \beta_{n}\left\|x_{n}-\tilde{x}\right\|^{2}+\left(1-\beta_{n}\right)\left\|(\gamma I+(1-\gamma) S) T_{\lambda_{n}} y_{n}-\tilde{x}\right\|^{2} \\
& \leq \beta_{n}\left\|x_{n}-\tilde{x}\right\|^{2}+\left(1-\beta_{n}\right)\left\|T_{\lambda_{n}} y_{n}-\tilde{x}\right\|^{2} \\
& =\beta_{n}\left\|x_{n}-\tilde{x}\right\|^{2}+\left(1-\beta_{n}\right)\left\|T_{\lambda_{n}} y_{n}-T_{\lambda_{n}}\left(\tilde{x}-\lambda_{n} A \tilde{x}\right)\right\|^{2} \\
& \leq \beta_{n}\left\|x_{n}-\tilde{x}\right\|^{2}+\left(1-\beta_{n}\right)\left\|y_{n}-\left(\tilde{x}-\lambda_{n} A \tilde{x}\right)\right\|^{2} \\
& =\beta_{n}\left\|x_{n}-\tilde{x}\right\|^{2}+\left(1-\beta_{n}\right)\left\|\alpha_{n} u_{n}+\left(1-\alpha_{n}\right) x_{n}-\lambda_{n} A x_{n}-\left(\tilde{x}-\lambda_{n} A \tilde{x}\right)\right\|^{2} \\
& =\left(1-\beta_{n}\right)\left\|\left(1-\alpha_{n}\right)\left(\left(x_{n}-\frac{\lambda_{n}}{1-\alpha_{n}} A x_{n}\right)-\left(\tilde{x}-\frac{\lambda_{n}}{1-\alpha_{n}} A \tilde{x}\right)\right)+\alpha_{n}\left(u_{n}-\tilde{x}\right)\right\|^{2} \\
& +\beta_{n}\left\|x_{n}-\tilde{x}\right\|^{2} \\
& =\left(1-\beta_{n}\right)\left(\left(1-\alpha_{n}\right)^{2}\left\|\left(x_{n}-\frac{\lambda_{n}}{1-\alpha_{n}} A x_{n}\right)-\left(\tilde{x}-\frac{\lambda_{n}}{1-\alpha_{n}} A \tilde{x}\right)\right\|^{2}\right. \\
& +2 \alpha_{n}\left(1-\alpha_{n}\right)\left\langle u_{n}-\tilde{x},\left(x_{n}-\frac{\lambda_{n}}{1-\alpha_{n}} A x_{n}\right)-\left(\tilde{x}-\frac{\lambda_{n}}{1-\alpha_{n}} A \tilde{x}\right)\right\rangle \\
& \left.+\alpha_{n}^{2}\left\|u_{n}-\tilde{x}\right\|^{2}\right)+\beta_{n}\left\|x_{n}-\tilde{x}\right\|^{2} \\
& \leq \beta_{n}\left\|x_{n}-\tilde{x}\right\|^{2}+\left(1-\beta_{n}\right)\left(\left(1-\alpha_{n}\right)^{2}\left\|x_{n}-\tilde{x}\right\|^{2}\right. \\
& \left.+2 \alpha_{n}\left(1-\alpha_{n}\right)\left\langle u_{n}-\tilde{x}, x_{n}-\frac{\lambda_{n}}{1-\alpha_{n}}\left(A x_{n}-A \tilde{x}\right)-\tilde{x}\right\rangle+\alpha_{n}^{2}\left\|u_{n}-\tilde{x}\right\|^{2}\right) \\
& \leq\left(1-\left(1-\beta_{n}\right) \alpha_{n}\right)\left\|x_{n}-\tilde{x}\right\|^{2} \\
& +\left(1-\beta_{n}\right) \alpha_{n}\left\{2\left(1-\alpha_{n}\right)\left\langle u_{n}-\tilde{x}, v_{n}-\tilde{x}\right\rangle+\alpha_{n}\left\|u_{n}-\tilde{x}\right\|^{2}\right\} .
\end{aligned}
$$

It is clear that $\sum_{n=1}^{\infty}\left(1-\beta_{n}\right) \alpha_{n}=\infty$ and $\lim \sup _{n \rightarrow \infty}\left(2\left(1-\alpha_{n}\right)\left\langle u_{n}-\tilde{x}, v_{n}-\tilde{x}\right\rangle+\alpha_{n} \| u_{n}-\right.$ $\left.\tilde{x} \|^{2}\right) \leq 0$. We can therefore apply Lemma 2.7 to conclude that $x_{n} \rightarrow \tilde{x}$. This completes the proof.

Corollary 3.2 Let $C$ be a nonempty closed convex subset of a real Hilbert space $H$ and let $F$ : $C \times C \rightarrow R$ be a bifunction satisfying conditions (H1)-(H4). Let $A: C \rightarrow H$ be an $\alpha$-inversestrongly monotone mapping and let $S: C \rightarrow C$ be a nonexpansive mapping. Suppose that $F(S) \cap M E P \neq \emptyset$. Let $x_{0} \in C,\left\{z_{n}\right\}$ and $\left\{x_{n}\right\}$ be sequences in $C$ generated by

$$
\left\{\begin{array}{l}
F\left(z_{n}, y\right)+\left\langle A x_{n}, y-z_{n}\right\rangle+\frac{1}{\lambda_{n}}\left\langle y-z_{n}, z_{n}-\left(\alpha_{n} u_{n}+\left(1-\alpha_{n}\right) x_{n}\right)\right\rangle \geq 0, \quad \forall y \in C \\
x_{n+1}=\beta_{n} x_{n}+\left(1-\beta_{n}\right) \gamma z_{n}+\left(1-\beta_{n}\right)(1-\gamma) S z_{n}
\end{array}\right.
$$

for all $n \geq 0$, where $\left\{\lambda_{n}\right\} \subset(0,2 \alpha),\left\{\alpha_{n}\right\} \subset(0,1)$ and $\left\{\beta_{n}\right\} \subset(0,1)$ satisfy

(r1) $\lim _{n \rightarrow \infty} u_{n}=u$ for some $u \in H$;

(r2) $\lim _{n \rightarrow \infty} \alpha_{n}=0$ and $\sum_{n=1}^{\infty} \alpha_{n}=\infty$;

(r3) $0<c \leq \beta_{n} \leq d<1$ and $\gamma \in(0,1)$;

(r4) $a\left(1-\alpha_{n}\right) \leq \lambda_{n} \leq b\left(1-\alpha_{n}\right)$, where $[a, b] \subset(0,2 \alpha)$ and $\lim _{n \rightarrow \infty}\left(\lambda_{n+1}-\lambda_{n}\right)=0$.

Then $\left\{x_{n}\right\}$ generated by (3.12) converges strongly to $\operatorname{Proj}_{F(S) \cap M E P}(u)$. 
Corollary 3.3 Let $C$ be a nonempty closed convex subset of a real Hilbert space $H$ and let $F: C \times C \rightarrow R$ be a bifunction satisfying conditions (H1)-(H4). Let $S: C \rightarrow C$ be a $\kappa-$ strictly pseudo-contractive mapping. Suppose that $F(S) \cap E P \neq \emptyset$. Let $x_{0} \in C,\left\{z_{n}\right\}$ and $\left\{x_{n}\right\}$ be sequences in $C$ generated by

$$
\left\{\begin{array}{l}
F\left(z_{n}, y\right)+\frac{1}{\lambda_{n}}\left\langle y-z_{n}, z_{n}-\left(\alpha_{n} u_{n}+\left(1-\alpha_{n}\right) x_{n}\right)\right\rangle \geq 0, \quad \forall y \in C, \\
x_{n+1}=\beta_{n} x_{n}+\left(1-\beta_{n}\right) \gamma z_{n}+\left(1-\beta_{n}\right)(1-\gamma) S z_{n}
\end{array}\right.
$$

for all $n \geq 0$, where $\left\{\lambda_{n}\right\} \subset(0,1),\left\{\alpha_{n}\right\} \subset(0,1)$ and $\left\{\beta_{n}\right\} \subset(0,1)$ satisfy

(r1) $\lim _{n \rightarrow \infty} u_{n}=u$ for some $u \in H$;

(r2) $\lim _{n \rightarrow \infty} \alpha_{n}=0$ and $\sum_{n=1}^{\infty} \alpha_{n}=\infty$;

(r3) $0<c \leq \beta_{n} \leq d<1$ and $\gamma \in[\kappa, 1)$;

(r4) $a\left(1-\alpha_{n}\right) \leq \lambda_{n} \leq b\left(1-\alpha_{n}\right)$, where $[a, b] \subset(0,1)$ and $\lim _{n \rightarrow \infty}\left(\lambda_{n+1}-\lambda_{n}\right)=0$.

Then $\left\{x_{n}\right\}$ generated by (3.13) converges strongly to $\operatorname{Proj}_{F(S) \cap E P}(u)$.

Corollary 3.4 Let $C$ be a nonempty closed convex subset of a real Hilbert space $H$ and let $F: C \times C \rightarrow R$ be a bifunction satisfying conditions (H1)-(H4). Let $S: C \rightarrow C$ be a nonexpansive mapping. Suppose that $F(S) \cap E P \neq \emptyset$. Let $x_{0} \in C,\left\{z_{n}\right\}$ and $\left\{x_{n}\right\}$ be sequences in $C$ generated by

$$
\left\{\begin{array}{l}
F\left(z_{n}, y\right)+\frac{1}{\lambda_{n}}\left\langle y-z_{n}, z_{n}-\left(\alpha_{n} u_{n}+\left(1-\alpha_{n}\right) x_{n}\right)\right\rangle \geq 0, \quad \forall y \in C, \\
x_{n+1}=\beta_{n} x_{n}+\left(1-\beta_{n}\right) \gamma z_{n}+\left(1-\beta_{n}\right)(1-\gamma) S z_{n}
\end{array}\right.
$$

for all $n \geq 0$, where $\left\{\lambda_{n}\right\} \subset(0,1),\left\{\alpha_{n}\right\} \subset(0,1)$ and $\left\{\beta_{n}\right\} \subset(0,1)$ satisfy

(r1) $\lim _{n \rightarrow \infty} u_{n}=u$ for some $u \in H$;

(r2) $\lim _{n \rightarrow \infty} \alpha_{n}=0$ and $\sum_{n=1}^{\infty} \alpha_{n}=\infty$;

(r3) $0<c \leq \beta_{n} \leq d<1$ and $\gamma \in(0,1)$;

(r4) $a\left(1-\alpha_{n}\right) \leq \lambda_{n} \leq b\left(1-\alpha_{n}\right)$, where $[a, b] \subset(0,1)$ and $\lim _{n \rightarrow \infty}\left(\lambda_{n+1}-\lambda_{n}\right)=0$.

Then $\left\{x_{n}\right\}$ generated by (3.14) converges strongly to $\operatorname{Proj}_{F(S) \cap E P}(u)$.

Corollary 3.5 Let $C$ be a nonempty closed convex subset of a real Hilbert space $H$ and let $F$ : $C \times C \rightarrow R$ be a bifunction satisfying conditions (H1)-(H4). Let $A: C \rightarrow H$ be an $\alpha$-inversestrongly monotone mapping and let $S: C \rightarrow C$ be a $\kappa$-strictly pseudo-contractive mapping. Suppose that $F(S) \cap M E P \neq \emptyset$. Let $x_{0} \in C,\left\{z_{n}\right\}$ and $\left\{x_{n}\right\}$ be sequences in $C$ generated by

$$
\left\{\begin{array}{l}
F\left(z_{n}, y\right)+\left\langle A x_{n}, y-z_{n}\right\rangle+\frac{1}{\lambda_{n}}\left\langle y-z_{n}, z_{n}-\left(1-\alpha_{n}\right) x_{n}\right\rangle \geq 0, \quad \forall y \in C, \\
x_{n+1}=\beta_{n} x_{n}+\left(1-\beta_{n}\right) \gamma z_{n}+\left(1-\beta_{n}\right)(1-\gamma) S z_{n}
\end{array}\right.
$$

for all $n \geq 0$, where $\left\{\lambda_{n}\right\} \subset(0,2 \alpha),\left\{\alpha_{n}\right\} \subset(0,1)$ and $\left\{\beta_{n}\right\} \subset(0,1)$ satisfy

(r2) $\lim _{n \rightarrow \infty} \alpha_{n}=0$ and $\sum_{n=1}^{\infty} \alpha_{n}=\infty$;

(r3) $0<c \leq \beta_{n} \leq d<1$ and $\gamma \in[\kappa, 1)$;

(r4) $a\left(1-\alpha_{n}\right) \leq \lambda_{n} \leq b\left(1-\alpha_{n}\right)$, where $[a, b] \subset(0,2 \alpha)$ and $\lim _{n \rightarrow \infty}\left(\lambda_{n+1}-\lambda_{n}\right)=0$.

Then $\left\{x_{n}\right\}$ generated by (3.15) converges strongly to $\operatorname{Proj}_{F(S) \cap M E P}(0)$, which is the minimum norm element in $F(S) \cap M E P$. 
Corollary 3.6 Let $C$ be a nonempty closed convex subset of a real Hilbert space $H$ and let $F: C \times C \rightarrow R$ be a bifunction satisfying conditions $(\mathrm{H} 1)-(\mathrm{H} 4)$. Let $S: C \rightarrow C$ be a $\kappa$ strictly pseudo-contractive mapping. Suppose that $F(S) \cap E P \neq \emptyset$. Let $x_{0} \in C,\left\{z_{n}\right\}$ and $\left\{x_{n}\right\}$ be sequences in $C$ generated by

$$
\left\{\begin{array}{l}
F\left(z_{n}, y\right)+\frac{1}{\lambda_{n}}\left\langle y-z_{n}, z_{n}-\left(1-\alpha_{n}\right) x_{n}\right\rangle \geq 0, \quad \forall y \in C, \\
x_{n+1}=\beta_{n} x_{n}+\left(1-\beta_{n}\right) \gamma z_{n}+\left(1-\beta_{n}\right)(1-\gamma) S z_{n}
\end{array}\right.
$$

for all $n \geq 0$, where $\left\{\lambda_{n}\right\} \subset(0,1),\left\{\alpha_{n}\right\} \subset(0,1)$ and $\left\{\beta_{n}\right\} \subset(0,1)$ satisfy

(r2) $\lim _{n \rightarrow \infty} \alpha_{n}=0$ and $\sum_{n=1}^{\infty} \alpha_{n}=\infty$;

(r3) $0<c \leq \beta_{n} \leq d<1$ and $\gamma \in[\kappa, 1)$;

(r4) $a\left(1-\alpha_{n}\right) \leq \lambda_{n} \leq b\left(1-\alpha_{n}\right)$, where $[a, b] \subset(0,1)$ and $\lim _{n \rightarrow \infty}\left(\lambda_{n+1}-\lambda_{n}\right)=0$.

Then $\left\{x_{n}\right\}$ generated by (3.16) converges strongly to $\operatorname{Proj}_{F(S) \cap E P}(0)$, which is the minimum norm element in $F(S) \cap E P$.

Corollary 3.7 Let $C$ be a nonempty closed convex subset of a real Hilbert space $H$ and let $F: C \times C \rightarrow R$ be a bifunction satisfying conditions (H1)-(H4). Let $A: C \rightarrow H$ be an $\alpha$-inverse-strongly monotone mapping. Suppose that MEP $\neq \emptyset$. Let $x_{0} \in C,\left\{z_{n}\right\}$ and $\left\{x_{n}\right\}$ be sequences in $C$ generated by

$$
\left\{\begin{array}{l}
F\left(z_{n}, y\right)+\left\langle A x_{n}, y-z_{n}\right\rangle+\frac{1}{\lambda_{n}}\left\langle y-z_{n}, z_{n}-\left(1-\alpha_{n}\right) x_{n}\right\rangle \geq 0, \quad \forall y \in C, \\
x_{n+1}=\beta_{n} x_{n}+\left(1-\beta_{n}\right) z_{n}
\end{array}\right.
$$

for all $n \geq 0$, where $\left\{\lambda_{n}\right\} \subset(0,2 \alpha),\left\{\alpha_{n}\right\} \subset(0,1)$ and $\left\{\beta_{n}\right\} \subset(0,1)$ satisfy

(r2) $\lim _{n \rightarrow \infty} \alpha_{n}=0$ and $\sum_{n=1}^{\infty} \alpha_{n}=\infty$;

(r3) $0<c \leq \beta_{n} \leq d<1$;

(r4) $a\left(1-\alpha_{n}\right) \leq \lambda_{n} \leq b\left(1-\alpha_{n}\right)$, where $[a, b] \subset(0,2 \alpha)$ and $\lim _{n \rightarrow \infty}\left(\lambda_{n+1}-\lambda_{n}\right)=0$.

Then $\left\{x_{n}\right\}$ generated by (3.17) converges strongly to $\operatorname{Proj}_{M E P}(0)$, which is the minimum norm element in MEP.

Corollary 3.8 Let $C$ be a nonempty closed convex subset of a real Hilbert space $H$ and let $F: C \times C \rightarrow R$ be a bifunction satisfying conditions (H1)-(H4). Suppose EP $\neq \emptyset$. Let $x_{0} \in C$, $\left\{z_{n}\right\}$ and $\left\{x_{n}\right\}$ be sequences in $C$ generated by

$$
\left\{\begin{array}{l}
F\left(z_{n}, y\right)+\frac{1}{\lambda_{n}}\left\langle y-z_{n}, z_{n}-\left(1-\alpha_{n}\right) x_{n}\right\rangle \geq 0, \quad \forall y \in C, \\
x_{n+1}=\beta_{n} x_{n}+\left(1-\beta_{n}\right) z_{n}
\end{array}\right.
$$

for all $n \geq 0$, where $\left\{\lambda_{n}\right\} \subset(0,1),\left\{\alpha_{n}\right\} \subset(0,1)$ and $\left\{\beta_{n}\right\} \subset(0,1)$ satisfy

(r2) $\lim _{n \rightarrow \infty} \alpha_{n}=0$ and $\sum_{n=1}^{\infty} \alpha_{n}=\infty$;

(r3) $0<c \leq \beta_{n} \leq d<1$;

(r4) $a\left(1-\alpha_{n}\right) \leq \lambda_{n} \leq b\left(1-\alpha_{n}\right)$, where $[a, b] \subset(0,1)$ and $\lim _{n \rightarrow \infty}\left(\lambda_{n+1}-\lambda_{n}\right)=0$.

Then $\left\{x_{n}\right\}$ generated by (3.18) converges strongly to $\operatorname{Proj}_{E P}(0)$, which is the minimum norm element in EP. 


\section{Competing interests}

The authors declare that they have no competing interests.

\section{Authors' contributions}

All authors read and approved the final manuscript.

\section{Author details}

'Department of Mathematics, Tianjin Polytechnic University, Tianjin, 300387, China. ${ }^{2}$ Department of Information Management, Cheng Shiu University, Kaohsiung, 833, Taiwan. ${ }^{3}$ Department of Mathematics and the RINS, Gyeongsang National University, Jinju, 660-701, Korea.

\section{Received: 25 February 2013 Accepted: 28 June 2013 Published: 12 July 2013}

\section{References}

1. Blum, E, Oettli, W: From optimization and variational inequalities to equilibrium problems. Math. Stud. $63,123-145$ (1994)

2. Ceng, LC, Al-Homidan, S, Ansari, QH, Yao, JC: An iterative scheme for equilibrium problems and fixed point problems of strict pseudo-contraction mappings. J. Comput. Appl. Math. 223, 967-974 (2009)

3. Ceng, LC, Schaible, S, Yao, JC: Implicit iteration scheme with perturbed mapping for equilibrium problems and fixed point problems of finitely many nonexpansive mappings. J. Optim. Theory Appl. 139, 403-418 (2008)

4. Chuang, CS, Lin, LJ, Takahashi, W: Halpern's type iterations with perturbations in Hilbert spaces: equilibrium solutions and fixed points. J. Glob. Optim. (2013). doi:10.1007/s10898-012-9911-6

5. Colao, V, Acedo, GL, Marino, G: An implicit method for finding common solutions of variational inequalities and systems of equilibrium problems and fixed points of infinite family of nonexpansive mappings. Nonlinear Anal. 71, 2708-2715 (2009)

6. Colao, V, Marino, G, Xu, HK: An iterative method for finding common solutions of equilibrium and fixed point problems. J. Math. Anal. Appl. 344, 340-352 (2008)

7. Combettes, PL, Hirstoaga, A: Equilibrium programming in Hilbert spaces. J. Nonlinear Convex Anal. 6, 117-136 (2005)

8. Konnov, IV, Schaible, S, Yao, JC: Combined relaxation method for mixed equilibrium problems. J. Optim. Theory Appl. 126, 309-322 (2005)

9. Moudafi, A: Weak convergence theorems for nonexpansive mappings and equilibrium problems. J. Nonlinear Convex Anal. 9, 37-43 (2008)

10. Moudafi, A, Théra, M: Proximal and dynamical approaches to equilibrium problems. In: Lecture Notes in Economics and Mathematical Systems, vol. 477, pp. 187-201. Springer, Berlin (1999)

11. Peng, JW, Yao, JC: A new hybrid-extragradient method for generalized mixed equilibrium problems, fixed point problems and variational inequality problems. Taiwan. J. Math. 12, 1401-1432 (2008)

12. Plubtieng, S, Punpaeng, R: A general iterative method for equilibrium problems and fixed point problems in Hilbert spaces. J. Math. Anal. Appl. 336, 455-469 (2007)

13. Qin, $X, C h o, S Y, K a n g, S M:$ Some results on generalized equilibrium problems involving a family of nonexpansive mappings. Appl. Math. Comput. 217, 3113-3126 (2010)

14. Qin, X, Cho, YJ, Kang, SM: Viscosity approximation methods for generalized equilibrium problems and fixed point problems with applications. Nonlinear Anal. 72, 99-112 (2010)

15. Takahashi, S, Takahashi, W: Strong convergence theorem for a generalized equilibrium problem and a nonexpansive mapping in a Hilbert space. Nonlinear Anal. 69, 1025-1033 (2008)

16. Xu, HK: An iterative approach to quadratic optimization. J. Optim. Theory Appl. 116, 659-678 (2003)

17. Yao, Y, Liou, YC: Composite algorithms for minimization over the solutions of equilibrium problems and fixed point problems. Abstr. Appl. Anal. 2010, Article ID 763506 (2010)

18. Yao, Y, Liou, YC, Yao, JC: Convergence theorem for equilibrium problems and fixed point problems of infinite family of nonexpansive mappings. Fixed Point Theory Appl. 2007, Article ID 64363 (2007)

19. Yao, Y, Shahzad, N: New methods with perturbations for non-expansive mappings in Hilbert spaces. Fixed Point Theory Appl. 2011, 79 (2011)

20. Zegeye, H, Ofoedu, EU, Shahzad, N: Convergence theorems for equilibrium problem, variational inequality problem and countably infinite relatively quasi-nonexpansive mappings. Appl. Math. Comput. 216, 3439-3449 (2010)

21. Zegeye, $\mathrm{H}$, Shahzad, N: A hybrid scheme for finite families of equilibrium, variational inequality and fixed point problems. Nonlinear Anal. 74, 263-272 (2011)

22. Zhang, SS, Joseph, HWL, Chan, CK: Algorithms of common solutions for quasi variational inclusion and fixed point problems. Appl. Math. Mech. 29, 571-581 (2008)

23. Suzuki, $\mathrm{T}$ : Strong convergence theorems for infinite families of nonexpansive mappings in general Banach spaces, Fixed Point Theory Appl. 2005, 103-123 (2005)

24. Zhou, $\mathrm{H}$ : Convergence theorems of fixed points for $\lambda$-strict pseudo-contractions in Hilbert spaces. Nonlinear Anal. 69, 456-462 (2008)

25. Marino, $\mathrm{C}, \mathrm{Xu}, \mathrm{HK}$ : Weak and strong convergence theorems for strict pseudocontractions in Hilbert spaces. J. Math. Anal. Appl. 329, 336-349 (2007)

doi:10.1186/1687-1812-2013-183

Cite this article as: Yao et al.: An iterative approach to mixed equilibrium problems and fixed points problems. Fixed Point Theory and Applications 2013 2013:183. 